\title{
Journal of

\section{Depth of Field: Teaching the Art of Reflection to Medical Students}

\author{
Gabrielle Brand*, Sandra Carr and Christopher Etherton-Beer \\ Faculty of Medicine, Dentistry and Health Sciences at the University of Western Australia, Australia
}

*Corresponding author: Gabrielle Brand, Lecturer in Health Professional Education, Education Centre, The University of Western Australia, Faculty of Medicine, Dentistry and Health Sciences, 35 Stirling Hwy, Crawley WA 6009, Australia, Tel: +6186488 6893; Fax: +61864886879; E-mail: Gabrielle.Brand@uwa.edu.au

Rec Date: Nov 01, 2016, Acc Date: Nov 10, 2016, Pub Date: Nov 12, 2016

Copyright: @ 2016 Brand G, et al. This is an open-access article distributed under the terms of the creative commons attribution license, which permits unrestricted use, distribution, and reproduction in any medium, provided the original author and source are credited.

Citation: Brand G, Carr S, Etherton-Beer C (2016) Depth of Field: Teaching the Art of Reflection to Medical Students . J Gerontol Geriatr Res 5: 361. doi: 10.4172/2167-7182.1000361

\section{Commentary}

Fostering reflective practice and lifelong learning skills is a core component of medical education, particularly during the clinical years. However, reflective models tend to focus on reflection-in-action and after-action, and neglect to consider reflection-before-action or as we term it 'preflection'. In addition, a growing ageing population and complex health care systems require medical educators to prepare future doctors to adopt positive approaches to older age and illness, including practicing humanistic models of care for older people. A recent focus on integrating arts and humanities-based pedagogy to complement traditional, empirically-driven medical curricula has been used to activate reflective processes in students. Hence, there is a need to include educational interventions that stimulate in-depth discussion, surface unexplored stereotypes and challenge medical students through reflection to widen their lens and consider new perspectives around ageing.

We developed depth of field: Exploring ageing (Hereby: DOF) a coproduced (with older adults) preflective learning resource to prompt and explore medical student's perceptions towards older people prior to their first geriatric medicine clinical placement. 128 second year medical students volunteered to participate in a 1 hour DOF session. Pre-post questionnaire and individual written reflections were collected to explore whether the documentary style photographs and collaborative classroom dialogue fosters reflective learning and has the potential to shift medical student's attitudes towards caring for older adults. The data showed that visual and narrative methodologies (used in DOF) stimulated reflective learning processes that generated a positive shift in medical students' perceptions towards older adults [1].

This DOF reflective learning methodology heightened an awareness and integration of medical students' thoughts (cognitive) and emotions (heart) by bringing perceptions of any potential hidden bias towards older adults into clearer focus. Creating the opportunities for reflection promoted personal and professional meaning making, a key element in augmenting transformational learning where shifts in perspectives occur. This active learning approach helped students envision working with older adults, created the tension required to challenge and dismantle misinformed assumptions which ultimately led to deeper insights and understandings of the lived experiences of older people. This included an exploration of how unconscious bias may influence the way they care for older people in the future. Future longitudinal studies are required to measure the impact of this type of preflective learning on medical students' subsequent clinical practice and interactions with older adults. However, we believe that medical education should place a greater emphasis on reflective activities including visual and narrative learning methodologies which we have learnt offers an innovative, cost effective and valuable approach to preparing medical students to care for older adults. Our future goal is to adapt this reflective learning approach for inclusion into teaching other specialities that care for stigmatized groups, including mental and indigenous health.

\section{Funding}

The authors disclose receipt of the following financial support for the research project. Funding was received from the Department of Health: Western Australia Clinical Training Network.

\section{References}

1. Brand G, Osborne A, Carroll M, Carr S, Etherton-Beer C (2016) Do photographs, older adults' narratives and collaborative dialogue foster anticipatory reflection ("preflection") in medical students? In Press. BMC Med Ed. 